\title{
FITOSSOCIOLOGIA E ANÁLISE COMPARATIVA DA COMPOSIÇÃO FLORÍSTICA DO CERRADO DA FLORA DE PARAOPEBA-MG ${ }^{1}$
}

Alexander Paulo do Carmo Balduino ${ }^{2}$, Agostinho Lopes de Souza ${ }^{3}$, João Augusto Alves Meira Neto ${ }^{4}$, Alexandre Francisco da Silva ${ }^{5}$ e Manoel Cláudio da Silva Júnior ${ }^{6}$

\begin{abstract}
RESUMO - Foi conduzido um estudo fitossociológico no Cerrado sentido restrito da Floresta Nacional de Paraopeba, comparando-se os resultados (qualitativos) com os de outras amostras. Foram estabelecidas 10 parcelas de 0,1 ha $(20 \times 50 \mathrm{~m})$, sendo incluídas no estudo todas as árvores $\left(\mathrm{C}_{30} \geq 15,7 \mathrm{~cm}\right)$. Foram registrados 1.990 indivíduos/ha (densidade total) e $18,13 \mathrm{~m}^{2} / \mathrm{ha}$ (área basal total). Os valores de riqueza (73 espécies/ 38 famílias), de diversidade Shannon $(3,57)$ e de uniformidade $(0,83)$ indicaram grande heterogeneidade e baixa dominânica ecológica. Leguminosae e Vochysiaceae foram as famílias mais ricas em espécies. O grupo das árvores mortas alcançou o maior valor de importância, seguido por Qualea parviflora, Pera glabrata, Erythroxylum suberosum e Qualea grandiflora. A análise comparativa de composições florísticas de 11 cerrados indicou uma forte influência da flora de Floresta Atlântica em Cerrado marginal do interior de São Paulo. Houve influência da localização geográfica na similaridade do Cerrado de Palmas, TO, com as outras áreas.
\end{abstract}

Palavras-chave: Cerrado sentido restrito, Fitossociologia, Floresta Nacional de Paraopeba e Similaridade.

\section{PHYTOSOCIOLOGICAL COMPOSITION AND FLORISTIC COMPARISON OF CERRADO OF PARAOPEBA FLORA - MG}

\begin{abstract}
A phytosociological study in the Cerrado "sensu strictu" of The Paraopeba Nacional Forest$M G$ was carried out, comparing the qualitative results with other samples. The Paraopeba National Forest suffered intense clearing in 1952, which motivated the phytosociological study of 1 hectare of woody vegetation and floristic comparison with others samples. Ten plots of $0.1 \mathrm{ha}(20 \times 50 \mathrm{~m})$ were established and included in the study all trees with $C_{30} \geq 15.7 \mathrm{~cm}$. A total of 1990 individuals $/$ ha (total density) and $18.13 \mathrm{~m}^{2} / \mathrm{ha}$ (total basal area) were recorded. The values of richness ( 73 species/38 families), of Shannon diversity (3.57) and the evenness (0.83) suggested great heterogeneity and low ecological dominance. Leguminosae and Vochysiaceae were the richest families in species. The group of dead trees reached the greater value of importance followed by Qualea parviflora, Pera glabrata, Erythroxylum suberosum and Qualea grandiflora. The floristic comparative analysis showed that the Atlantic Forest influenced the similarity values between Santa Bárbara's Cerrado and the others. It was different for the Cerrado of Palmas, in which geographic distance was shown to be the most important factor influencing the floristic composition.
\end{abstract}

Key words: Cerrado stricto sensu, Similarity, Phytosociology, Natonal Forest (FLONA) of Paraopeba

\footnotetext{
${ }^{1}$ Recebido em 06.10.2003 e aceito para publicação em 25.11.2004.

${ }^{2}$ Consultor Técnico Ambiental -SQS 307 BLOCOGAPT305-Asa Sul-Brasília(DF).CEP70354-070.<alexander.paulo@terra.com.br>.

${ }^{3}$ Departamento de Engenharia Florestal - Universidade Federal de Viçosa (MG). <alsouza@.ufv.br>.

${ }^{4}$ Departamento de Biologia Vegetal - Universidade Federal de Viçosa (MG).<j.meira@ufv.br>. <afsilva@ufv.br>.

${ }^{5}$ Departamento de Engenharia Florestal - Universidade de Brasília (DF).<mcsj@unb.br>.
} 


\section{INTRODUÇÃO}

O Cerrado é uma das 25 áreas do mundo consideradas críticas para conservação, devido à riqueza biológica e à alta pressão antrópica a que vem sendo submetido (MMA, 2002). Dos aproximadamente dois milhões de quilômetros quadrados iniciais $(25 \%$ do território nacional) restam, hoje, cerca de 350.000 (MITTERMEIER et al., 1999). Fisionomicamente, mesmo quando não é perturbado por fogo, corte e pastoreio, ocorre em todas as alturas e densidades da camada lenhosa, de floresta fechada, em todas as formas intermediárias, até campo limpo graminoso (EINTEN, 2001).

Sua fisionomia mais comum é o Cerrado sentido restrito, formação savânica caracterizada pela presença de árvores baixas, inclinadas, tortuosas, com ramificações irregulares e retorcidas, e geralmente com evidência de queimadas (RIBEIRO e WALTER, 1998). Sua flora é composta por dois grupos de espécies: aquelas da camada lenhosa, que varia de 3-5 m de altura, com cobertura arbórea de 10 a $60 \%$; e as próprias da camada rasteira, com predominância de gramíneas (EINTEN, 2001; FELFILI et al., 2002).

Estudos relativos à florística e à fitossocilogia de Cerrado sentido restrito (RIZZINI, 1963; HERINGER etal., 1977; RIBEIROetal., 1985; FELFILIeSILVAJÚNIOR, 1992; MARIMON et al., 1998; NUNES, 2001; ANDRADE et al., 2002) têm apontado um bom nível de conhecimento sobre essa vegetação, entretanto trabalhos voltados para uma abordagem comparativa entre amostras de diferentes localidades do bioma evidenciam carência de dados, diante da distribuição espacial de espécies, refletida na grande variabilidade de mosaicos (FELFILI et al., 1993; FELFILI et al., 1994; CASTRO, 1994; RATTER et al., 2000).

O Cerrado sentido restrito, por ocupar terrenos planos de solos profundos, que são propícios à agricultura mecanizável, está desaparecendo, tendo em vista as boas condições físicas do solo para abrigar construções civis e outras atividades antrópicas (FELFILI et al., 2002).

A região de Paraopeba, exemplo típico do modelo citado anteriormente, têm grande parte da cobertura natural remanescente concentrada em sua Floresta Nacional, importante unidade de conservação da região, representante da flora regional nativa.
O objetivo do trabalho foi descrever a composição fitossociológica do Cerrado sentido restrito da Floresta Nacional de Paraopeba e comparar floristicamente esse Cerrado com outros do Domínio do Cerrado.

\section{MATERIAL E MÉTODO}

\section{1. Área de estudo}

O estudo foi realizado na Floresta Nacional de Paraopeba-MG (FLONA-Paraopeba), nas coordenadas geográficas de $19^{\circ} 20^{\prime} \mathrm{S}$ de latitude e $44^{\circ} 20^{\prime} \mathrm{W}$ de longitude, com uma altitude variável de 734 m.s.m. ao sul, a 750 m.s.m. ao norte, distando $90 \mathrm{~km}$ de Belo Horizonte e $625 \mathrm{~km}$ de Brasília, pela rodovia BR-040, Brasília-Rio de Janeiro. Aárea total da FLONAé de aproximadamente 200 ha, estando totalmente cercada e dividida por aceiros, que delimitam 59 talhões (Figura 1). O clima da região foi caracterizado como subtropical úmido, com verão chuvoso e estação seca de abril a setembro (THORNTHWAITE e MATHER, 1957), e os solos foram classificados como Latossolo Vermelho-Escuro, Latossolo Vermelho-Amarelo e Latossolo-Amarelo (THIBAU et al., 1975). O histórico da área relata constantes intervenções externas, por parte dos "minhoqueiros", cuja atividade resulta em solo completamente escavado e revolvido e em plantas herbáceas e lenhosas provenientes da regeneração natural completamente removida. Segundo Silva Júnior (1984), há ainda registro de fogo (1960 e 1963) e de desmatamento total em 200 ha da vegetação local (1952), circundada por Mata Seca (sobre afloramento de calcário) e por Cerradão. A vegetação é classificada fitofisionomicamente como Cerrado sentido restrito (GOODLAND, 1971).

\subsection{Amostragem da vegetação arbórea}

Foram alocadas 10 parcelas aleatórias de 0,1 ha (20 x 50 m) (Figura 1), conforme método estabelecido pelo Projeto Biogeografia do Bioma Cerrado (FELFILI et al., 1994). Nessa ocasião, foram medidos os diâmetros (à altura de 0,30 m do solo, DA30) e identificados todos os indivíduos lenhosos e palmeiras com circunferência mínima de 15,7 cm (diâmetro de $5 \mathrm{~cm}$ ). O material desconhecido foi coletado e identificado por meio de literatura especializada e por comparação no Herbário do Departamento de Biologia Vegetal da Universidade Federal de Viçosa (VIC). 


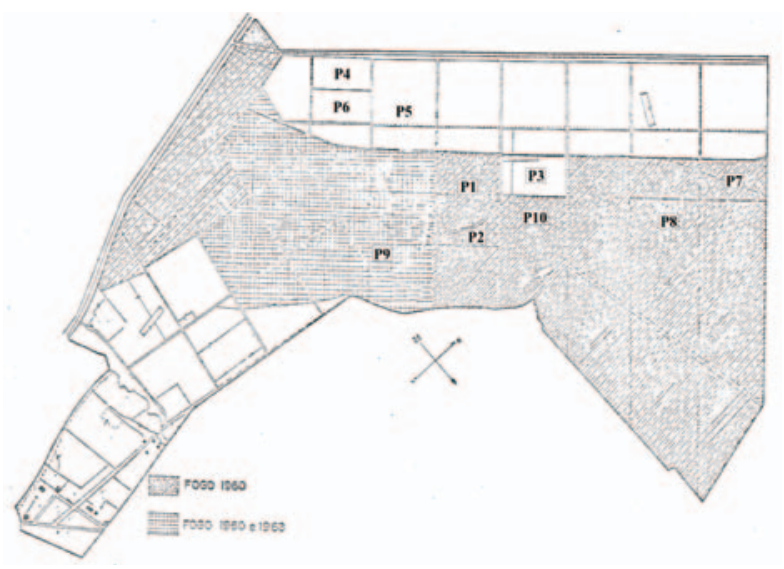

Figura 1 - Croqui da Floresta Nacional de Paraopeba, MG, com a localização das parcelas amostradas (P1 a P10).

Figure 1 - Outline of the Paraopeba Nacional Forest, $M G$, with the localization of sampled plots (P1 to P10).

\subsection{Fitossociologia e composição florística}

As famílias e espécies amostradas foram organizadas, utilizando-se o sistema de classificação de Cronquist (1981), à exceção da família Leguminosae. Os nomes dos táxons foram atualizados em Mendonça et al. (1998) e no "site" www.mobot.org/w3t/search/ vast.html (MISSOURI BOTANICAL GARDEN, 2003). Foram estimados no "Software" Microsoft Excel 2000: riqueza, diversidade de Shannon, uniformidade de Pielou e parâmetros fitossociológicos usuais em trabalhos de fitossociologia (MUELLER-DOMBOIS e ELLENBERG, 1974).
O trecho de Cerrado sentido restrito estudado foi comparado qualitativamente (presença e ausência de espécies) com os citados em outros trabalhos (Quadro 1), utilizando-se o índice de similaridade de Jaccard (BROWER e ZAR, 1984), transformado em medida de distância (GOWER, 1967). Para as interpretações florísticas, foram empregados três algoritmos (agrupamentos por médias não-ponderadas, UPGMA; ligação simples; e ligação completa) (SNEATHe SOKAL, 1973), sendo os diagramas de ligação obtidos no software Statistica/W5.0.

\section{RESULTADOS E DISCUSSÃO}

As estimativas dos parâmetros relativos à fitossociologia são apresentadas no Quadro 2. Foram registradas a densidade total de 1990 indivíduos/ha e a área basal total de $18,1388 \mathrm{~m}^{2} / \mathrm{ha}$, valores acima daqueles referentes a outras localidades de Cerrado sentido restrito. Na Chapada Pratinha (GO, MG e DF) houve variação de 664 a 1.396 indivíduos e de 5,79 a $10,76 \mathrm{~m}^{2} / \mathrm{ha}$ (FELFILI et al., 1994); na Chapada do Espigão Mestre do São Francisco (MG e BA), de 628 a 835 indivíduos e de 6,19 a $8,89 \mathrm{~m}^{2} / \mathrm{ha} \mathrm{(FELFILI} \mathrm{et}$ al., 2001); e no Distrito Federal, mediana de 1.042,8 indivíduos/ha de $7,48 \mathrm{~m}^{2} / \mathrm{ha}$.

Os valores de riqueza (73 espécies e 38 famílias), de diversidade $(3,57)$ e de uniformidade $(0,83)$ foram considerados altos, indicando grande heterogeneidade e baixa dominância ecológica. Esses valores estão dentro do intervalo de variação encontrado para o DF, Chapada Pratinha e Chapada do Espigão Mestre do São Francisco (FELFILI e SILVA-JÚNIOR, 1992; FELFILI et al., 1993; FELFILI et al., 1994; PIRES et al., 2002).

Quadro 1 - Trabalhos de Cerrado sentido restrito consultados para comparação florística com o presente estudo Table 1 - Papers of Cerrado sensu stricto consulted for floristic comparison with the present study

\begin{tabular}{lccc}
\hline \multicolumn{1}{c}{ Referência } & Local & Método & Critério de Inclusão \\
\hline Silva Júnior (1984) & Paraopeba, MG & Ponto quadrante & DAS $\geq 5 \mathrm{~cm}$ \\
Meira Neto (1991) & Santa Bárbara, SP & Parcela & DAS $\geq 3 \mathrm{~cm}$ \\
Santos (2000) & Palmas, TO & Parcela & CAS $\geq 10 \mathrm{~cm}$ \\
Felfili et al. (1994) & Parque Nacional de Brasília, DF & Parcela & DA30 $\geq 5 \mathrm{~cm}$ \\
Felfili et al. (1994) & Área de Proteção Ambiental Cabeça de Veado, DF & Parcela & DA30 $\geq 5 \mathrm{~cm}$ \\
Felfili et al. (1994) & Estação Ecológica Águas Emendadas, DF & Parcela & DA30 $\geq 5 \mathrm{~cm}$ \\
Felfili et al. (1994) & Silvânia, GO & Parcela & DA30 $\geq 5 \mathrm{~cm}$ \\
Felfili et al. (1994) & Paracatu, MG & Parcela & DA30 $\geq 5 \mathrm{~cm}$ \\
Felfili et al. (1994) & Patrocínio, MG & Parcela & DA30 $\geq 5 \mathrm{~cm}$ \\
Silva (1993) & Uberlândia, MG & Parcela & DAP $\geq 3,8 \mathrm{~cm}$ \\
\hline
\end{tabular}

* DAS (diâmetro à altura do solo), CAS (circunferência à altura do solo), DA30 (diâmetro à altura de 0,30 m do solo), e DAP (diâmetro à altura de $1,30 \mathrm{~m}$ do solo). 
Quadro 2 - Espécies e parâmetros fitossociológicos do Cerrado sentido restrito da FLONA Paraopeba (MG) Table 2 - Species and phytosociological parameters of the Cerrado stricto sensu of the FLONA Paraopeba (MG)

\begin{tabular}{|c|c|c|c|c|c|c|c|}
\hline Espécies & Famílias & $\mathrm{N}$ & $\mathrm{AB}$ & DR & DoR & FR & VI \\
\hline$\overline{\text { Mortas em pé }}$ & - & 177 & 1,7218 & 8,89 & 9,49 & 2,83 & $\overline{21,22}$ \\
\hline Qualea parviflora Mart. & Vochysiaceae & 184 & 1,5155 & 9,25 & 8,36 & 2,83 & 20,43 \\
\hline Pera glabrata (Schott) Baill. & Euphorbiaceae & 111 & 1,2627 & 5,58 & 6,96 & 2,83 & 15,37 \\
\hline Erythroxylum suberosum A. St.-Hil. & Erythroxylaceae & 127 & 0,7645 & 6,38 & 4,21 & 2,83 & 13,43 \\
\hline Qualea grandiflora Mart. & Vochysiaceae & 89 & 0,7054 & 4,47 & 3,89 & 2,83 & 11,19 \\
\hline Erythroxylum daphnites Mart. & Erythroxylaceae & 77 & 0,6942 & 3,87 & 3,83 & 2,83 & 10,53 \\
\hline Kielmeyera cf. grandiflora (Wawra) Saddi & Clusiaceae & 95 & 0,4867 & 4,77 & 2,68 & 2,55 & 10,01 \\
\hline Curatella americana $\mathrm{L}$. & Dilleniaceae & 60 & 0,7319 & 3,02 & 4,04 & 2,83 & 9,88 \\
\hline Eugenia dysenterica DC. & Myrtaceae & 73 & 0,6522 & 3,67 & 3,60 & 2,55 & 9,81 \\
\hline Xylopia aromatica (Lam.) Mart. & Annonaceae & 69 & 0,6035 & 3,47 & 3,33 & 2,27 & 9,06 \\
\hline Machaerium opacum Vogel & Leguminosae & 53 & 0,6715 & 2,66 & 3,70 & 2,55 & 8,91 \\
\hline Salvertia convallariaeodora A. St.-Hil. & Vochysiaceae & 40 & 0,9255 & 2,01 & 5,10 & 1,70 & 8,81 \\
\hline Byrsonima verbascifolia (L.) Rich. ex A. Juss. & Malpighiaceae & 67 & 0,4078 & 3,37 & 2,25 & 2,55 & 8,16 \\
\hline Acosmium dasycarpum (Vogel) Yakovlev & Leguminosae & 43 & 0,3690 & 2,16 & 2,03 & 2,55 & 6,74 \\
\hline Annona crassiflora Mart. & Annonaceae & 26 & 0,3963 & 1,31 & 2,18 & 2,83 & 6,32 \\
\hline Roupala montana Aubl. & Proteaceae & 43 & 0,3525 & 2,16 & 1,94 & 1,98 & 6,09 \\
\hline Hyptis cana Pohl ex Benth. & Lamiaceae & 45 & 0,3290 & 2,26 & 1,81 & 1,70 & 5,77 \\
\hline Magonia pubescens A. St.-Hil. & Sapindaceae & 56 & 0,4323 & 2,81 & 2,38 & 0,28 & 5,48 \\
\hline Plathymenia reticulata Benth. & Leguminosae & 24 & 0,4632 & 1,21 & 2,55 & 1,70 & 5,46 \\
\hline Byrsonima coccolobifolia H. B. K. & Malpighiaceae & 30 & 0,2492 & 1,51 & 1,37 & 2,27 & 5,15 \\
\hline Rudgea viburnoides (Cham.) Benth. & Rubiaceae & 33 & 0,2060 & 1,66 & 1,14 & 2,27 & 5,06 \\
\hline Caryocar brasiliense Cambess. & Caryocaraceae & 14 & 0,4244 & 0,70 & 2,34 & 1,98 & 5,03 \\
\hline Terminalia argentea Mart. \& Zucc. & Combretaceae & 25 & 0,3239 & 1,26 & 1,79 & 1,70 & 4,74 \\
\hline Erythroxylum deciduum A. St.-Hil. & Erythroxylaceae & 25 & 0,1359 & 1,26 & 0,75 & 2,55 & 4,56 \\
\hline Dimorphandra mollis Benth. & Leguminosae & 22 & 0,2452 & 1,11 & 1,35 & 1,98 & 4,44 \\
\hline Piptocarpha rotundifolia (Less.) Baker & Asteraceae & 30 & 0,2167 & 1,51 & 1,19 & 1,70 & 4,40 \\
\hline Schefflera macrocarpa (Seem.) Frodin & Araliaceae & 27 & 0,1320 & 1,36 & 0,73 & 2,27 & 4,35 \\
\hline Tapirira guianensis Aubl. & Anacardiaceae & 13 & 0,2926 & 0,65 & 1,61 & 1,98 & 4,25 \\
\hline Myrcia lingua Berg & Myrtaceae & 27 & 0,1300 & 1,36 & 0,72 & 1,98 & 4,06 \\
\hline Bowdichia virgilioides $\mathrm{H}$. B. K. & Leguminosae & 15 & 0,2789 & 0,75 & 1,54 & 1,70 & 3,99 \\
\hline Alibertia sessilis (Vell.) K. Schum. & Rubiaceae & 25 & 0,1405 & 1,26 & 0,77 & 1,70 & 3,73 \\
\hline Stryphnodendron adstringens (Mart.) Coville & Leguminosae & 20 & 0,1037 & 1,01 & 0,57 & 1,98 & 3,56 \\
\hline Styrax camporum Pohl & Styracaceae & 23 & 0,1459 & 1,16 & 0,80 & 1,42 & 3,38 \\
\hline Zeyheria digitalis (Vell.) Hoehne \& Kuhlm. & Bignoniaceae & 14 & 0,0711 & 0,70 & 0,39 & 1,98 & 3,08 \\
\hline Diospyros híspida A. DC. & Ebenaceae & 27 & 0,2228 & 1,36 & 1,23 & 0,28 & 2,87 \\
\hline Guapira noxia (Netto) Lundell & Nyctaginaceae & 11 & 0,1450 & 0,55 & 0,80 & 1,42 & 2,77 \\
\hline Erythroxylum tortuosum Mart. & Erythroxylaceae & 11 & 0,0452 & 0,55 & 0,25 & 1,70 & 2,50 \\
\hline Sclerolobium paniculatum Vogel & Leguminosae & 10 & 0,0866 & 0,50 & 0,48 & 1,42 & 2,40 \\
\hline Hymenaea stigonocarpa Mart. & Leguminosae & 7 & 0,0945 & 0,35 & 0,52 & 1,42 & 2,29 \\
\hline Qualea multiflora Mart. & Vochysiaceae & 12 & 0,0387 & 0,60 & 0,21 & 1,42 & 2,23 \\
\hline Astronium fraxinifolium Schott & Anacardiaceae & 9 & 0,0768 & 0,45 & 0,42 & 1,13 & 2,01 \\
\hline Myrsine guianensis (Aubl.) Kuntze & Myrsinaceae & 11 & 0,0479 & 0,55 & 0,26 & 1,13 & 1,95 \\
\hline Symplocos nitens (Pohl) Benth. & Symplocaceae & 4 & 0,0836 & 0,20 & 0,46 & 1,13 & 1,79 \\
\hline Tabebuia aurea ( Silva Manso) & Bignoniaceae & 5 & 0,0635 & 0,25 & 0,35 & 0,85 & 1,45 \\
\hline Benth. \& Hook.f. ex S. Moore & & & & & & & \\
\hline Agonandra brasiliensis Benth. \& Hook. f. & Opiliaceae & 7 & 0,0312 & 0,35 & 0,17 & 0,85 & 1,37 \\
\hline Siparuna guianensis Aubl. & Monimiaceae & 6 & 0,0248 & 0,30 & 0,14 & 0,85 & 1,29 \\
\hline Enterolobium gummiferum (Mart.) J. F. Macbr. & Leguminosae & 5 & 0,0321 & 0,25 & 0,18 & 0,85 & 1,28 \\
\hline Miconia albicans (Sw.) Triana & Melastomataceae & 5 & 0,0182 & 0,25 & 0,10 & 0,85 & 1,20 \\
\hline Aspidosperma tomentosum Mart. & Apocynaceae & 8 & 0,0421 & 0,40 & 0,23 & 0,57 & 1,20 \\
\hline Strychnos pseudoquina A. St.-Hil. & Loganiaceae & 3 & 0,0810 & 0,15 & 0,45 & 0,57 & 1,16 \\
\hline Palicourea rigida Kunth & Rubiaceae & 4 & 0,0124 & 0,20 & 0,07 & 0,85 & 1,12 \\
\hline Myrcia cf. formosiana DC. & Myrtaceae & 3 & 0,0119 & 0,15 & 0,07 & 0,85 & 1,07 \\
\hline Tabebuia ochracea (Cham.) Standl. & Bignoniaceae & 3 & 0,0400 & 0,15 & 0,22 & 0,57 & 0,94 \\
\hline Myrcia cf. tomentosa (Aubl.) DC. & Myrtaceae & 4 & 0,0187 & 0,20 & 0,10 & 0,57 & 0,87 \\
\hline Vochysia rufa Mart. & Vochysiaceae & 4 & 0,0110 & 0,20 & 0,06 & 0,57 & 0,83 \\
\hline Qualea cordata Spreng. & Vochysiaceae & 2 & 0,0227 & 0,10 & 0,13 & 0,57 & 0,79 \\
\hline Protium heptaphyllum (Aubl.) Marchand & Burseraceae & 2 & 0,0724 & 0,10 & 0,40 & 0,28 & 0,78 \\
\hline Syagrus flexuosa (Mart.) Becc. & Arecaceae & 3 & 0,0382 & 0,15 & 0,21 & 0,28 & 0,64 \\
\hline
\end{tabular}


Quadro 2 - cont.

Table 2 - cont.

\begin{tabular}{|c|c|c|c|c|c|c|c|}
\hline Espécies & Famílias & $\mathrm{N}$ & $\mathrm{AB}$ & DR & DoR & FR & VI \\
\hline Platypodium elegans Vogel & Leguminosae & 2 & 0,0459 & 0,10 & 0,25 & 0,28 & 0,64 \\
\hline Austroplenckia populnea (Reissek) Lundell & Celastraceae & 1 & 0,0418 & 0,05 & 0,23 & 0,28 & 0,56 \\
\hline Tibouchina sp. & Melastomataceae & 3 & 0,0168 & 0,15 & 0,09 & 0,28 & 0,53 \\
\hline Copaifera langsdorffii Desf. & Leguminosae & 2 & 0,0249 & 0,10 & 0,14 & 0,28 & 0,52 \\
\hline Ouratea spectabilis (Mart.) Engl. & Ochnaceae & 2 & 0,0115 & 0,10 & 0,06 & 0,28 & 0,45 \\
\hline Connarus suberosus Planch. & Connaraceae & 2 & 0,0094 & 0,10 & 0,05 & 0,28 & 0,44 \\
\hline Vanillosmopsis cf. erythropappa $\mathrm{Sch}$. Bip. & Asteraceae & 1 & 0,0147 & 0,05 & 0,08 & 0,28 & 0,41 \\
\hline Tabebuia sp. & Bignoniaceae & 1 & 0,0097 & 0,05 & 0,05 & 0,28 & 0,39 \\
\hline Antonia ovata Pohl & Loganiaceae & 1 & 0,0035 & 0,05 & 0,02 & 0,28 & 0,35 \\
\hline Miconia cf. ligustroides (DC.) Naudin & Melastoma & 1 & 0,00 & 0,05 & 0,02 & 0,28 & 0,35 \\
\hline $\begin{array}{l}\text { Pseudobombax longiflorum } \\
\text { (Mart. \& Zucc.) A. Robyns }\end{array}$ & Bombacaceae & 1 & 0,0032 & 0,05 & 0,02 & 0,28 & 0,35 \\
\hline Baccharis sp. & Ast & 1 & & 0,0 & 0,0 & 0,28 & 0,35 \\
\hline Dalbergia miscolobium Benth. & Legum & 1 & 0,0 & 0,0 & 0, & 0,28 & 0,35 \\
\hline Tocoyena formosa (Cham. \& Schltdl.) K. Schum. & Rubiaceae & 1 & 0,0022 & 0,0 & 0,01 & 0,28 & 0,35 \\
\hline Heteropterys byrsonimifolia A. Juss. & Malpighiaceae & 1 & 0,0020 & 0,0 & 0,01 & 0,28 & 0,34 \\
\hline Lafoensia pacari A. St.-Hil. & Lythraceae & 1 & 0,0020 & 0,05 & 0,01 & 0,28 & 0,34 \\
\hline Total & & 1990 & 18,1388 & 100 & 100 & 100 & 300 \\
\hline
\end{tabular}

* Espécies ordenadas de acordo com os valores de importância. ** $\mathrm{N}$ (número de indivíduos/ha), AB (área basal - m²/ha), DR (densidade relativa $\%$ ), DoR ( dominância relativa - \%), FR (freqüência relativa - \%) e VI (valor de importância $=$ DR + DoR + FR).

As famílias Leguminosae e Vochysiaceae contribuíram com 12 e 6 espécies, respectivamente (Quadro 2), seguidas por Asteraceae (4 sp), Bignoniaceae (4 sp), Erythroxylaceae (4 sp), Rubiaceae (4 sp), Myrtaceae (4 sp), Melastomataceae (3 sp), Malpighiaceae (3 sp), Anacardiaceae (2 sp), Annonaceae (2 sp) e Loganiaceae ( $2 \mathrm{sp}$ ). As famílias restantes contribuíram com apenas uma espécie cada. Para a flora fanerogâmica do Bioma Cerrado, Mendonça et al. (1998) apontaram Leguminosae, Asteraceae e Poaceae (Gramineae) como sendo as famílias mais ricas. Em se tratando da flora lenhosa de Cerrado sentido restrito, famílias como Leguminosae, Vochysiaceae, Myrtaceae, Malpighiaceae e Rubiaceae comumente apresentam altos valores de riqueza florística (RIZZINI, 1963; CASTRO, 1994; FELFILI et al., 1994; RATTER et al., 2000; NUNES, 2001).

A análise do Quadro 2 indica que, na maioria das vezes, o parâmetro que mais contribuiu para a determinação da importância de uma espécie foi a densidade relativa; algumas vezes, entretanto, a dominância relativa apareceu como o parâmetro mais importante, como no caso das espécies Pera glabrata, Curatella americana, Salvertia convallariaeodora, Caryocar brasiliense e Bowdichia virgilioides, que apresentam indivíduos de maiores tamanhos. A frequiência relativa teve menor influência na ordenação do VI, fato justificado em parte pelo número e tamanho utilizados para o estabelecimento das unidades amostrais. Sua maior influência foi no posicionamento de Magonia pubescens e Diospyros hispida, espécies com altos valores de densidade e área basal, concentradas em apenas uma unidade amostral.

O grupo principal, formado pelas mortas em pé, Qualea parviflora, Pera glabrata, Erythroxylum suberosum, Qualea grandiflora, Erythroxylum daphnites, Kielmeyera cf. grandiflora, Curatella americana, Eugenia dysenterica e Xylopia aromatica (Quadro 2), somou 43,65\% do VI total. Desse grupo, Qualea parviflora e Qualea grandiflora, citadas como típicas acumuladoras de alumínio (HARIDASAN, 1982; MEDEIROS, 1983; HARIDASAN, 2000), têm sempre ocupado posições superiores de importância em comunidades de Cerrado sentido restrito em solos distróficos (SILVA JÚNIOR, 1984; FELFILI e SILVA JÚNIOR, 1992; FELFILI et al., 1994; FELFILI et al., 2001; ANDRADE et al., 2002; SILVA et al., 2002).

Os índices de similaridade (Quadro 3) variaram de 0,77 (Parque Nacional de Brasília - DF e Área de Proteção Ambiental Cabeça de Veado-DF) a 0,12 (Santa Bárbara-SP e Palmas - TO). Seguindo-se o procedimento de Kent e Coker (1992), que trata valores iguais ou superiores a 0,5 como altos, a similaridade foi tida como baixa, na maioria dos valores apresentados.

R. Árvore, Viçosa-MG, v.29, n.1, p.25-34, 2005 
Quadro 3 - Valores de Similaridade entre as amostras comparadas

Table 3 - Similarity values between compared samples

\begin{tabular}{|c|c|c|c|c|c|c|c|c|c|c|c|}
\hline & A & B & $\mathrm{C}$ & $\mathrm{D}$ & $E$ & $\mathrm{~F}$ & G & $\mathrm{H}$ & I & $\mathbf{J}$ & $\mathrm{K}$ \\
\hline A & 1,00 & & & & & & & & & & \\
\hline B & 0,56 & 1,00 & & & & & & & & & \\
\hline $\mathrm{C}$ & 0,24 & 0,18 & 1,00 & & & & & & & & \\
\hline D & 0,21 & 0,17 & 0,12 & 1,00 & & & & & & & \\
\hline $\mathrm{E}$ & 0,31 & 0,28 & 0,15 & 0,19 & 1,00 & & & & & & \\
\hline $\mathrm{F}$ & 0,29 & 0,29 & 0,15 & 0,23 & 0,77 & 1,00 & & & & & \\
\hline $\mathrm{G}$ & 0,35 & 0,36 & 0,18 & 0,21 & 0,61 & 0,65 & 1,00 & & & & \\
\hline $\mathrm{H}$ & 0,33 & 0,34 & 0,17 & 0,22 & 0,54 & 0,60 & 0,59 & 1,00 & & & \\
\hline I & 0,35 & 0,41 & 0,16 & 0,19 & 0,39 & 0,40 & 0,47 & 0,45 & 1,00 & & \\
\hline $\mathbf{J}$ & 0,28 & 0,31 & 0,21 & 0,20 & 0,46 & 0,50 & 0,50 & 0,42 & 0,46 & 1,00 & \\
\hline $\mathrm{K}$ & 0,25 & 0,24 & 0,20 & 0,14 & 0,25 & 0,27 & 0,30 & 0,32 & 0,30 & 0,28 & 1,00 \\
\hline
\end{tabular}

A-Paraopeba - MG (este trabalho); B - Paraopeba, MG (SILVA JÚNIOR, 1984); C-Santa Bárbara, SP(MEIRA NETO, 1991); D - Palmas, TO (SANTOS, 2000); E - Parque Nacional de Brasília, DF (FELFILI et al., 1994); F - Área de Proteção Ambiental Cabeça de Veado, DF (FELFILI et al., 1994); G - Estação Ecológica Águas Emendadas, DF (FELFILI et al., 1994); H - Silvânia, GO (FELFILI et al., 1994); I - Paracatu, MG (FELFILI et al., 1994); J - Patrocínio, MG (FELFILI et al., 1994); e K - Uberlândia, MG (SILVA, 1993).

A análise dos diagramas de distâncias de ligação representados pelas Figuras 2, 3 e 4 indica a formação de grupos consistentes que estão presentes nos três dendrogramas.

A formação dos grupos teve influência da localização geográfica das áreas e da distribuição de espécies consideradas peculiares e acessórias (RIZZINI, 1963; HERINGER et al., 1977; CASTRO, 1994). Casos extremos foram o Cerrado marginal de Santa Bárbara, SP, que teve as suas relações de similaridades mais influenciadas pela flora acessória de origem atlântica e o de Palmas, maior influência da distância geográfica, sendo a área mais distante das demais (Figuras 2, 3 e 4).

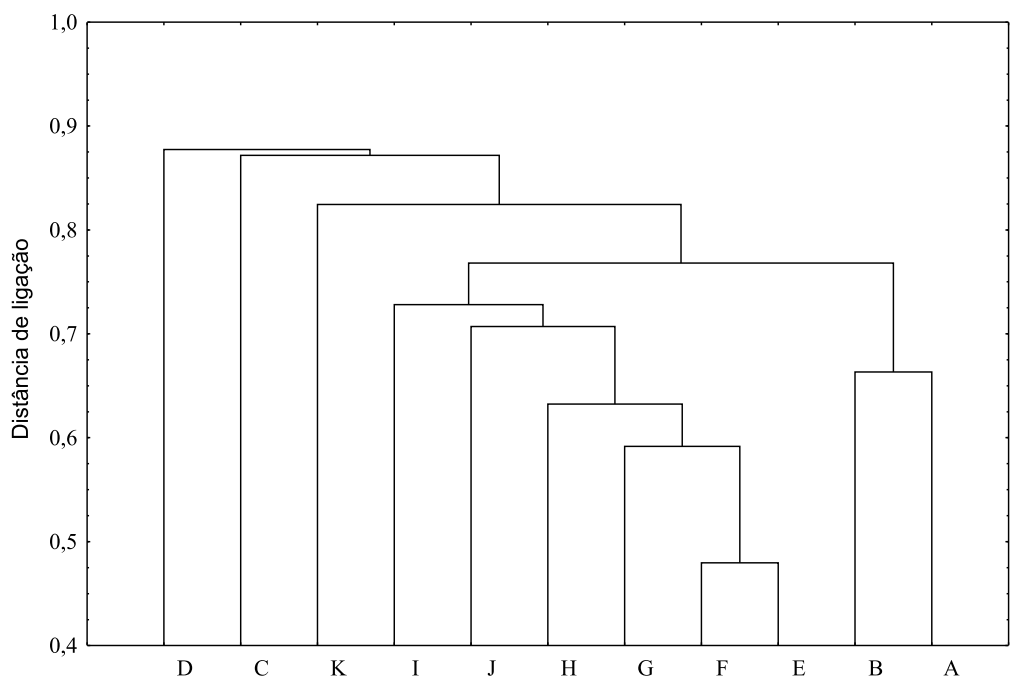

A - Paraopeba, MG (este trabalho); B - Paraopeba, MG (SILVA JÚNIOR, 1984); C - Santa Bárbara, SP(MEIRA NETO, 1991); D - Palmas, TO (SANTOS, 2000); E - Parque Nacional de Brasília, DF (FELFILI et al., 1994); F - Área de Proteção Ambiental Cabeça de Veado, DF (FELFILI et al., 1994); G - Estação Ecológica Águas Emendadas, DF (FELFILI et al., 1994); H - Silvânia, GO (FELFILI et al., 1994); I - Paracatu, MG (FELFILI et al., 1994); J - Patrocínio, MG (FELFILI et al., 1994); e K - Uberlândia, MG (SILVA, 1993).

Figura 2 - Diagrama obtido por ligação simples, representando as distâncias medidas entre as amostras comparadas. Figure 2 - Diagram obtained by simple linkage, representing the distances measured among compared samples.

R. Árvore, Viçosa-MG, v.29, n.1, p.25-34, 2005 


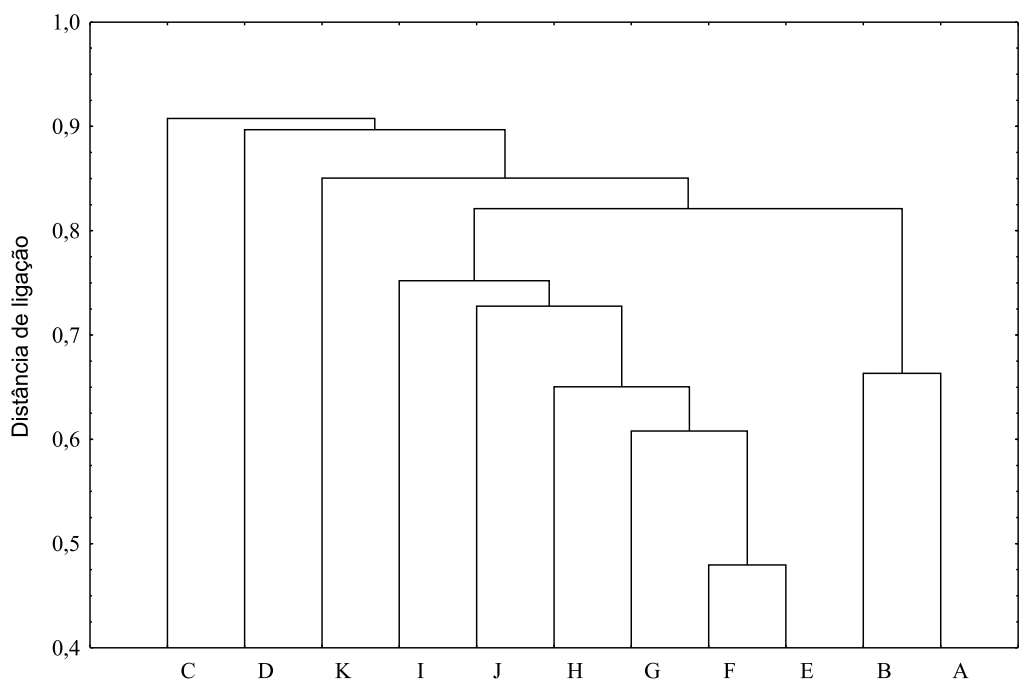

A - Paraopeba, MG (este trabalho); B - Paraopeba, MG (SILVA JÚNIOR, 1984); C - Santa Bárbara, SP (MEIRA NETO, 1991); D - Palmas, TO (SANTOS, 2000); E - Parque Nacional de Brasília, DF (FELFILI et al., 1994); F - Área de Proteção Ambiental Cabeça de Veado, DF (FELFILI et al., 1994); G - Estação Ecológica Águas Emendadas, DF (FELFILI et al., 1994); H - Silvânia, GO (FELFILI et al., 1994); I - Paracatu, MG (FELFILI et al., 1994); J - Patrocínio, MG (FELFILI et al., 1994); e K - Uberlândia, MG (SILVA, 1993).

Figura 3 - Diagrama obtido por UPGMA (médias não-ponderadas), representando as distâncias medidas entre as amostras comparadas.

Figure 3 - Diagram obtained by UPGMA (non ponderable averages), representing the distances measured among compared samples.

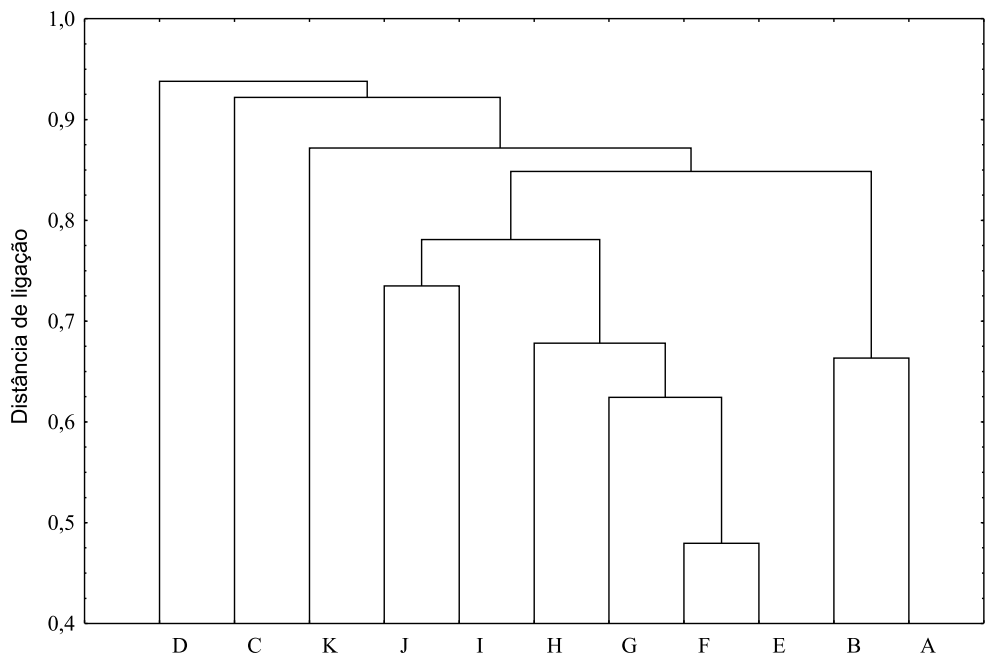

A - Paraopeba, MG (este trabalho); B - Paraopeba, MG (SILVA JÚNIOR, 1984); C - Santa Bárbara, SP(MEIRA NETO, 1991); D - Palmas, TO (SANTOS, 2000); E - Parque Nacional de Brasília, DF (FELFILI et al., 1994); F - Área de Proteção Ambiental Cabeça de Veado, DF (FELFILI et al., 1994); G - Estação Ecológica Águas Emendadas, DF (FELFILI et al., 1994); H - Silvânia, GO (FELFILI et al., 1994); I - Paracatu, MG (FELFILI et al., 1994); J - Patrocínio, MG (FELFILI et al., 1994); e K - Uberlândia, MG (SILVA, 1993).

Figura 4 - Diagrama obtido por ligação completa, representando as distâncias medidas entre as amostras comparadas. Figure 4 - Diagram obtained by complete linkage, representing the distances measured among compared samples. 
A alta heterogeneidade do componente lenhoso entre as áreas comparadas (Figuras 2, 3 e 4) corrobora os resultados citados em outros trabalhos, que mostram a existência de padrões fitogeográficos no bioma Cerrado, baseados na distribuição das suas espécies lenhosas (CASTRO, 1994; FELFILI et al., 1994; RATTER et al., 1996; RATTER et al., 2000; FELFILI et al., 2001). As diferenças encontradas indicam que as espécies do Cerrado sentido restrito se caracterizam por se distribuírem espacialmente em "mosaicos", sempre com uma combinação de menos de 100 espécies por área estudada, e mesmo as comunidades situadas em áreas próximas apresentam-se florística e estruturalmente diferenciadas (FELFILI et al., 1993).

\section{AGRADECIMENTOS}

Ao funcionário Ernani Alberto Rocha e à Jake Reis do Carmo, pela ajuda no trabalho de campo; À CAPES, ao Mestrado em Botânica da Universidade Federal de Viçosa e à Família Carmo, pelo apoio financeiro; À Dra. Aristéa Alves Azevedo, pela disponibilização de verbas para custear o trabalho de campo; ao chefe da FLONAParaopeba Agostinho Gomes da Fonseca, pela permissão para realização do trabalho; ao Engenheiro Florestal Henrique Cruvinel, pelas sugestões; e à Andréia Reis do Carmo, pela ajuda com o "abstract".

\section{REFERÊNCIAS BIBLIOGRÁFICAS}

ANDRADE, L. A.; FELFILI, J. M.; VIOLATTI, L. Fitossociologia de uma área de Cerrado denso na RECOR-IBGE, Brasília-DF. Acta Botânica Brasílica, v.16, n.2, p.225-240, 2002.

BROWER, J.E.; ZAR, J.H. Field and laboratory methods for general ecology. (2.ed.). Iowa: Wm. C. Brown Company, 1984. 226p.

CAstro, A. A . J. F. Comparação florístico-geográfica (Brasil) e fitossociológica (Piauí-São Paulo) de amostras de cerrado. 1994. 520f. Tese (Doutorado em Ciências Biológicas) Universidade Estadual de Campinas, Campinas, 1994.

CRONQUIST, A. An Integrated system of classification of flowering plants. New York: Columbia University, 1981. 1262p.
EINTEN, G. Vegetação natural do Distrito Federal. Brasília: SEBRAE/DF, 2001. 162p.

FELFILI, J.M.; SILVA JÚNIOR, M.C. Floristic composition, phytosociology and comparison of cerrado and gallery florests at Fazenda Água Limpa, Federal District, Brazil. In: FURLEY, P.A.; RATTER, J.A.; PROCTOR, J.A. (Eds.). Nature and dynamics of forest savanna boundaries. London: Chapman \& Hall, 1992. p.393-415.

FELFILI, J.M. et al. Análise comparativa da florística e fitossociologia da vegetação arbórea do cerrado sensu stricto na chapada Pratinha-DFBrasil. Acta Botanica Brasílica, v.6, n.2, p.27-46, 1993.

FELFILI, J.A. et al. Projeto biogeografia do bioma Cerrado: vegetação \& solos. Cadernos de Geociências, v.12, p.1-166. 1994.

FELFILI, J. M. et al. Fitossocilogia da vegetação arbórea. In: FELFILI, J. M.; SILVA JÚNIOR, M. C. Biogeografia do Bioma Cerrado: Estudo fitofisionômico na Chapada do Espigão Mestre do São Francisco. Brasília: Universidade de Brasília, Departamento de Engenharia Florestal, 2001. 152p.

FELfili, J. M. et al. Plantas da APA gama e cabeça de veado: espécies, ecossistemas e recuperação. Brasília: Universidade de Brasília, 2002. 52p.

GOODLAND, R. A. A physiognomic analysis of the "Cerrado" vegetation of Central Brazil. Journal of Ecology, v.59, p.411-419, 1971.

GOWER, J. C. A comparison of some methods of cluster analysis. Biometrics, v.23, p.623-637, 1967.

HARIDASAN, M. Aluminum accumulation by some cerrado vegetation native species of Central Brasil. Plant and Soil, v.65, p.265-273, 1982.

HARIDASAN, M. Nutrição mineral de plantas nativas do cerrado. Revista Brasileira de Fisiologia Vegetal, v.12, n.1, p.54-64, 2000.

HERINGER, E.P. et al. A flora do cerrado. In: FERRI, M.G. SIMPÓSIO SOBRE O CERRADO, 4., 1977, São Paulo. Anais... Universidade de São Paulo, 1977. p.15-36. 
KENT, M.; COKER, P. Vegetation description analyses. London: Behaven Press, 1992.363p.

MARIMON, B.S.; VARELLA, R.F.; JÚNIOR, B.H.M. Fitossociologia de uma área de cerrado de encosta em Nova Xavantina, Mato Grosso. Boletim do Herbário Ezechias Paulo Heringer, v.3, p.82-101, 1998.

MEDEIROS, R.A. Comparação do estado nutricional de algumas espécies acumuladoras e não acumuladoras de alumínio, nativas do cerrado. 1983. $91 \mathrm{f}$. Dissertação (Mestrado em Ciências Biológicas) Universidade de Brasília, Brasília,1983.

MEIRA NETO, J.A.A. Composição florística e fitossociologia de Vegetação de cerrado sensu lato da Estação Ecológica de Santa Bárbara (E.E.S.B.), município de Águas de Santa Bárbara, estado de São Paulo. 1991. 105f. Dissertação (Mestrado em Ciências Biológicas) Universidade de Campinas, Campinas, 1991.

MENDONÇA, R.C. et al. Flora vascular do cerrado. In: SANO, S.M.; ALMEIDA, S.P. (Eds.) Cerrado, ambiente e flora. Planaltina: Embrapa, 1998. p.289-556.

BRASIL. MINISTÉRIO DO MEIO AMBIENTE. Biodiversidade brasileira: avaliação e identificação de áreas prioritárias para conservação, utilização sustentável e repartição de benefícios da biodiversidade brasileira. Brasília: MMA/SBF, 2002. 404p.

MISSOURI BOTANICAL GARDEN. W3 Trópicos. (http://www.mobot.org/w3 search/ vast.html). 2003.

MITTERMEIER, R. A. et al. Hotspots: Earth's biologically richest and endangered terrestrial ecoregions. México: CEMEX, 1999. 431p.

MUELLER DOMBOIS, D.Y.; ELLENBERG, M. Aims and methods in vegetation ecology. New York: Willey \& Sons, 1974. 547p.

NUNES, R. V. Padrões de distribuição lenhosa do Cerrado sentido restrito do Distrito Federal. 2001. 44f. Dissertação (Mestrado em Ciências Biológicas) - Universidade de Brasília, Brasília, 2001.
PIRES, A.; FELFILI, J.M.; ABREU, A.R. Florística e fitossociologia do cerrado stricto sensu na APA de Cafuringa-DF. Boletim do Herbário Ezechias Paulo Heringer, v.4, p.5-20, 1999.

RATTER, J.A. et al. Estudo preliminar da distribuição das espécies lenhosas da fitofisionomia cerrado sentido restrito nos estados compreendidos pelo bioma Cerrado. Boletim do Herbário Esechias Paulo Heringer, v. 5. p.5-43, 2000.

RIBEIRO, J.F.; SILVA, J.C.S.; BATMANIAN, G.J. Fitossociologia de tipos fisionômicos de cerrado em Planaltina-DF. Revista Brasileira de Botânica, v.8, p.131-142, 1985.

RIBEIRO, J.F.; WALTER, B.M.T. 1998.

Fitofisionomias do bioma Cerrado. In: SANO, S.M.; ALMEIDA, S.P. (Eds.). Cerrado ambiente e flora. Planaltina: Embrapa, 1998. p.289-556.

RIZZINI, C.T. A flora do cerrado, análise florística das savanas Centrais. In: SIMPÓSIO

SOBRE O CERRADO. São Paulo: Universidade de São Paulo/ Edgard Blücher, 1963. p.125-177. 1963.

SANTOS, E.R. Análise florística e estrutura fitossociológica da vegetação lenhosa de um trecho de cerrado stricto sensu do Parque Estadual do Lajeado, Palmas-TO. 2000. 64f. Dissertação (Mestrado em Botânica) - Universidade Federal de Viçosa, Viçosa, 2000.

SILVA, G.M. Relações solo-vegetação como instrumento para o manejo da vegetação do cerrado no Triângulo Mineiro. 1993. 136f. Tese (Doutorado em Ciência Florestal) - Universidade Federal de Viçosa, Viçosa, 1992.

SILVA, L. et al. Levantamento florístico e fitossociológico em duas áreas de Cerrado sensu stricto no Parque Estadual de Caldas Novas, Goiás. Acta Botonica Brasileira, v.16, n.1, p.43-53, 2002.

R. Árvore, Viçosa-MG, v.29, n.1, p.25-34, 2005 
SILVA JÚNIOR, M.C. Composição florística, estrutura e parâmetros fitossociológicos do cerrado e sua relação com o solo na Estação Florestal de Experimentação de Paraopeba-MG. 1984. 130f. Dissertação (Mestrado em Ciência Florestal) - Universidade Federal de Viçosa, Viçosa, 1984.

SNEATH, P.H.; SOKAL, R.R. Numerical taxonomy. San Francisco: W.H. Freeman and Company, 1973. 573p.
THIBAU, C.E. et al. Inventário preliminar expedido na Estação Florestal de Experimentação de Paraopeba em Minas Gerais. Brasil Florestal, v.6, n.21, p.34-71, 1975.

THORNWAITE, C.W.; MATHER, J.R. Instructions and tables for computing potential evapotranspiration and water balance. Centerton: 1957. (Publication Climat, v.10, n.3). 